Nouvelles perspectives en sciences sociales

Revue internationale de systémique complexe et d'études relationnelles

\title{
Construire des sociétés artificielles pour comprendre les phénomènes sociaux réels
}

\section{Frédéric Amblard}

Volume 5, numéro 2, mai 2010

Sur le thème de la simulation

URI : https://id.erudit.org/iderudit/044076ar

DOI : https://doi.org/10.7202/044076ar

Aller au sommaire du numéro

Éditeur(s)

Prise de parole

ISSN

1712-8307 (imprimé)

1918-7475 (numérique)

Découvrir la revue

Citer cet article

Amblard, F. (2010). Construire des sociétés artificielles pour comprendre les phénomènes sociaux réels. Nouvelles perspectives en sciences sociales, 5(2), 69-77. https://doi.org/10.7202/044076ar
Résumé de l'article

Nous présentons ici rapidement l'approche de modélisation et de simulation multi-agent, ses principales caractéristiques ainsi que son intérêt pour les sciences sociales. En particulier, nous insistons sur la proximité de cette formalisation avec des cadres de pensées classiques en sciences sociales comme l'individualisme méthodologique et nous proposons un usage possible de cette approche comme outil permettant de formaliser et d'interroger les représentations des systèmes sociaux par le scientifique en sciences sociales. 


\title{
Construire des sociétés artificielles pour comprendre les phénomènes sociaux réels
}

\author{
FrÉdÉRIC AMBLARD \\ Université Toulouse \\ Institut de Recherche en Informatique de Toulouse (IRIT), \\ Université Paul Sabatier
}

T a construction de sociétés artificielles, c'est-à-dire de simuLlations informatiques dans lesquelles des entités, des personnages, adoptent des comportements sociaux, est souvent perçue soit en termes d'applications purement ludiques (les Sims en sont un bon exemple), soit comme une occasion offerte aux informaticiens de jouer à Dieu en construisant des mondes improbables. Cette approche peut, cependant, également être utilisée par le chercheur en sciences sociales pour interroger ses représentations du social par la formalisation de ces représentations et l'investigation des expérimentations qui y sont associées. C'est cette proposition que je tenterai de défendre ici.

On attribue souvent au prix Nobel Herbert Simon l'affirmation que les sciences sociales sont les vraies sciences « dures". De fait, les systèmes sociaux, en particulier les systèmes sociaux humains, sont composés d'entités hétérogènes, dont la rationalité est limitée (par calcul, par information, etc.) et dont les interactions peuvent être directes ou indirectes par l'entremise, par exemple, de modifications apportées à leur environnement. Dans 
ces systèmes, l'information est en grande partie locale et se diffuse avec déformation dans le système. Enfin, contrairement à une hypothèse encore trop souvent soutenue par l'économie orthodoxe du fait de son adhésion à la théorie walrasienne de l'équilibre général, l'équilibre n'est certainement pas une propriété des systèmes sociaux mais, au mieux, une tendance et, a minima sans doute, une représentation que certaines entités (certains économistes en particulier) se font du système auquel elles appartiennent.

L'étude de ces systèmes complexes soulève dès lors la question des outils et des méthodes à mettre en place afin de rendre compte de leurs propriétés principales dans l'objectif d'interroger et de comprendre les mécanismes sous-jacents producteurs des régularités observées de certains phénomènes collectifs. La simulation orientée agents a vocation de remplir ce rôle, en particulier pour ce qui concerne l'introspection des représentations du social construites notamment par le scientifique en sciences sociales.

Concernant la méthodologie générale associée à l'approche de simulation orientée agents, elle consiste, à partir d'un phénomène collectif que l'on cherche à étudier et à comprendre en définissant une question de modélisation et une ou des hypothèses explicatives concernant la production de ce phénomène, à décrire d'abord formellement les attributs et les comportements des entités qui constituent ce système, puis les interactions qui ont lieu entre ces entités. Il s'agira ensuite de décrire l'organisation de ces entités entre elles (par exemple, sous la forme d'un réseau social) et ses règles d'évolution ainsi que celles de l'environnement associé. Une fois ces éléments spécifiés formellement, le scientifique modélisateur a tout ce qu'il lui faut pour faire évoluer in silico un état du système donné correspondant à une situation particulière. Il reste donc au scientifique, expérimentateur cette fois, à déterminer ces conditions initiales, c'est-à-dire l'état du système sur lequel il veut tester les hypothèses de modélisation qu'il a proposées et formalisées. Après avoir déterminé les observables de sa simulation, c'est-à-dire les indicateurs qu’il 
souhaite suivre au cours de la simulation, le scientifique possède tous les éléments qui lui permettent de conduire ce que l'on pourrait définir comme une expérience de simulation. Une fois la simulation exécutée, la remise en perspective des hypothèses de départ et de la manière dont elles ont été formalisées au regard des résultats produits l'amène à réfléchir, d'une part, sur le modèle formalisé et la manière dont les résultats sont produits, c'est-à-dire le jeu fin de l'association des comportements et des interactions entre agents dans le modèle et, d'autre part, à interroger ses hypothèses au regard du phénomène qu'il recherche à étudier et à comprendre.

Les trois principales caractéristiques que l'on peut associer à cette approche de modélisation et qui la distinguent des approches de modélisation classiques sont, à mon sens, les suivantes.

1) Lapproche agent, en se focalisant particulièrement sur le comportement des entités constitutives du système, est congruente avec l'individualisme méthodologique qui est le cadre de pensée adopté pour la compréhension de la construction des phénomènes sociaux et elle est à même d'interroger les mécanismes microscopiques (l'échelle des individus), générateurs des régularités macroscopiques auxquelles s'intéresse le scientifique.

2) La simulation, en quittant sa forme précédente qui équivalait à l'exécution itérative d'un schéma de calcul numérique et surtout en remplaçant la sémantique classique de l'itération comme étape d'un schéma numérique par une représentation du déroulement du temps dans le système, permet dès lors à cette approche de formaliser des hypothèses dynamiques qui concernent l'évolution des entités et du système.

3) Enfin, la formalisation algorithmique associée à cette approche rend, par sa plus grande proximité avec la représentation que l'on peut se construire concernant la représentation d'un système social et de son évolution, la formalisation plus adéquate aux hypothèses que l'on se 
construit qu'un formalisme mathématique qui implique possiblement un changement de cadre de pensée lorsque l'on passe du raisonnement concernant le système au raisonnement concernant le modèle. Cela reste l'impression d'un informaticien, mais je pense que l'on peut rester dans le même cadre de pensée, manipuler les mêmes objets mentaux ou du moins des objets mentaux qui ont la même forme, et ce, que l'on raisonne sur le système que l'on cherche à étudier ou sur le modèle de ce système, sa formalisation algorithmique.

Cependant, cette richesse d'expression pour formaliser les mécanismes générateurs des phénomènes sociaux et potentiellement à même d'en interroger la complexité comporte un inconvénient majeur. En construisant un système artificiel qui capture la complexité du phénomène social, en associant les comportements hétérogènes de nombreuses entités en interaction à la production de phénomènes sociaux au niveau macroscopique, et malgré ce qui peut être vécu par le chercheur en sciences sociales comme des simplifications violentes, on ne réduit pas entièrement la complexité du système étudié. En effet, une fois le modèle formalisé et programmé, on a affaire à un système social, artificiel certes, composé d'entités hétérogènes en interactions. En réalisant des expériences de simulation sur ce système, on a éventuellement la possibilité d'observer des régularités qui semblent émerger de la simulation. Pour autant les mécanismes sous-jacents producteurs de ces régularités, l'intime entrelacement des comportements et des interactions n'est pas directement intelligible. Il s'agit alors, bien sûr, d'interroger le modèle, les simulations, pour comprendre comment les hypothèses telles qu'elles ont été formalisées ont produit les résultats en question et ceci principalement en jouant sur les observables de la simulation, par exemple en suivant dans le temps l'évolution du comportement d'un agent particulier. Plutôt que d'adopter l'attitude classique en modélisation qui consiste à interpréter les résultats du modèle directement par rapport au phénomène étudié, il s'agit bien plutôt ici de mettre en place une interpréta- 
tion à deux niveaux de ces résultats. Tout d'abord au niveau du modèle lui-même, la simulation étant déterministe, un mécanisme a produit ces résultats; il s'agit de l'identifier clairement, de construire une théorie du comportement du modèle, avant de réaliser une mise en perspective de cette théorie par rapport au phénomène réel étudié. Cette dernière comparaison est véritablement celle qui porte l'intérêt de la démarche puisqu'elle permet, une fois l'hypothèse formalisée et ses conséquences connues et théorisées, de questionner le phénomène réel étudié.

Dès lors, un tel outil peut être envisagé pour de nombreuses applications ou dans de nombreux cadres d'utilisation. L'un d'entre eux en particulier retient mon attention: il s'agit du modèle ou plus généralement du processus de modélisation comme artefact pour la réflexion. L'effort de formalisation des hypothèses implique un ensemble de clarifications et de désambiguïsations conceptuelles. Les concepts à retenir pour la modélisation étant les concepts pertinents et clairement définis, il s'agira ensuite de les articuler entre eux. Le chercheur modélisateur est ainsi amené à épurer son dispositif conceptuel pour ne garder que les concepts qui ont réellement du sens par rapport à la problématique étudiée; il est également amené à les préciser et à préciser le lien existant entre ces différents concepts. Cette tâche reste, bien sûr, limitée par l'expressivité du langage de formalisation utilisé qui est nécessairement plus faible que celle du discours en langue naturelle (son principal intérêt étant la non-ambiguïté).

Une utilisation des modèles de simulation congruente avec la précédente concerne l'utilisation de ces modèles comme des substrats pour l'aide à la projection d'hypothèses d'évolution portant sur un grand nombre d'entités en interaction. Lorsque l'on émet des hypothèses sur les comportements individuels d'une population, si l'on arrive encore passablement à imaginer ou à calculer les changements de comportements à l'échelle individuelle, il ne nous est pas possible (sous réserve d'hypothèses d'évolution même à peine évoluées) de réaliser ce calcul pour l'ensemble d'une population dont les individus interagissent les 
uns avec les autres, provoquant par ces interactions les changements de comportements au sein de la population. L'ordinateur via la simulation est précisément le support qui permettra d'exécuter ce calcul et de nous faire entrevoir la projection des hypothèses que l'on a faites au niveau individuel. Il s'agit alors de l'ordinateur considéré comme une grosse machine à calculer les évolutions d'un système dont nous sommes capables de donner les règles (on ne juge pas ici de la qualité de ces règles), mais dont nous ne sommes pas capables de calculer les effets produits par leur application sur le système.

Enfin, le fait de revenir sur le modèle, de l'interroger à l'issue de la simulation pour comprendre finement les mécanismes de production des résultats observés et de les mettre en perspective par rapport, à titre d'exemple, aux attentes que le chercheur modélisateur pouvait avoir au moment de la construction de son modèle, amène à envisager le modèle comme artefact d'accompagnement de la réflexion du chercheur quant à l'objet de ses recherches dont il construit une image qui en partage les grandes propriétés.

Dans un contexte un peu différent, qui est celui de l'aide à la décision par la modélisation ou par les modèles, on peut dire que là où les modèles mathématiques classiques permettent la prédiction ou la prévision (avec parfois les résultats que l'on sait), l'approche de simulation orientée-agent, dont la faiblesse est précisément le manque de fiabilité quantitative de ces résultats (puisque les mécanismes de production ne sont pas complètement contrôlés), peut proposer au décideur de comprendre en profondeur le système qu'il doit, par exemple, réguler; il s'agit donc plutôt d'anticipation (au sens où le modèle peut être vu comme un automate à produire des possibles) que d'un outil de prédiction aux résultats duquel on peut éventuellement se fier mais dont les mécanismes de production ne peuvent être compris ni par le décideur, ni par le modélisateur puisqu'ils ne sont pas prévus dans le modèle.

Ce positionnement du modèle comme artefact pour la réflexion s'inscrit dans un courant qui propose la reconstruction des 
phénomènes comme source de compréhension. La proposition initiale de Seymour Pappert avec le langage Logo et les dispositifs associés (on se souviendra du robot Tortue qui avait fait son apparition dans les écoles primaires françaises dans les années 1980) était de faire appréhender aux enfants la géométrie sous un aspect constructiviste par la mise à disposition d'un langage de programmation traduisant des opérations élémentaires de la géométrie (baisser/lever le crayon, tourner, avancer). Les enfants utilisant Logo appréhendaient la géométrie de manière différente et l'approche permettait de revisiter les concepts plus théoriques (telles les droites parallèles). C'est à sa suite que Michael Resnick propose, dans les années 1990, la plateforme de simulation multi-agent Starlogo pour appréhender les phénomènes distribués, en particulier en biologie, par leur reconstruction. L'élève cherche, en proposant des comportements à ses agents, à reproduire qualitativement un phénomène distribué (le tri du couvain ou la recherche de nourriture dans une fourmilière). Le schéma proposé ici est identique pour investiguer les phénomènes sociaux humains. Il s'agit, par une démarche de type expérimental par essai-erreur, de déterminer des règles de comportements individuels plausibles qui permettent de produire les phénomènes étudiés.

Cette approche amène, de fait, à poser quelques questions ou à délimiter quelques évolutions possibles. Évolution du rôle tenu par le modélisateur tout d'abord. Envisagé classiquement comme une compétence plutôt technique (si l'on considère la modélisation statistique) visant à analyser des données empiriques et à en tirer des informations pertinentes (tendances, corrélations, par exemple), le rôle proposé s'approche plutôt ici d'une activité d'expérimentateur, le modélisateur cherchant à déterminer un dispositif et des conditions expérimentales (la formalisation du modèle et les conditions initiales de la simulation) qui conduisent à la reproduction qualitative d'un phénomène donné.

Pour accompagner cette démarche, quelques points de positionnement restent ainsi à définir, en particulier par comparaison aux approches de modélisation classiques. Une approche métho- 
dologique tout d'abord. Dans le domaine de la simulation orientée-agents, les tenants de l'approche KISS (Keep It Simple, Stupid.) insistent sur le critère de parcimonie des modèles proposés, la plus simple " explication " possible d'un phénomène étant incidemment la meilleure ou du moins la plus probable. Les tenants de l'approche KIDS (Keep It Descriptive, Stupid.) défendent, quant à eux, une approche de modélisation qui, si elle correspond toujours à une abstraction (pour ne pas dire une simplification) du phénomène étudié, n'en reste pas moins homomorphique à celui-ci, mettant en jeu et manipulant des concepts et des objets de même forme que ceux du système étudié. La position raisonnable face à ces deux propositions consisterait à défendre une voie moyenne qui ne dépouille pas le modèle des éléments qui permettent d'interroger le phénomène étudié (en ce sens tenter l'homomorphie est plutôt raisonnable) tout en n'empêchant pas un des objectifs de l'approche qui est la construction d'une théorie explicative de l'émergence de phénomènes in silico, c'est-à-dire la production des résultats par les seules règles formalisées dans le modèle. Sur ce dernier point, cette théorie explicative est sans doute plus délicate à construire lorsque le nombre d'hypothèses contenues dans le modèle est important.

Concernant le positionnement par rapport aux approches de modélisation classiques, les difficultés rencontrées sont souvent liées à une incompréhension des différences entre les approches, différences qui tiennent à la fois au positionnement de la modélisation dans le projet de recherche et à l'utilisation du modèle. Le modèle multi-agent est envisagé davantage comme un outil permettant d'accompagner la réflexion du chercheur que comme l'aboutissement d'un projet de recherche. Un problème essentiel tient à l'évaluation des modèles. Si dans la démarche de modélisation classique, un critère définitif qui est souvent mis en avant - probablement à tort d'ailleurs si on suit la réflexion de Jean-Marie Legay, par exemple - concerne l'adéquation des résultats du modèle aux données empiriques, on sent bien que ce critère a du mal à s'appliquer seul pour l'évaluation de la 
démarche de modélisation/simulation multi-agent pour laquelle ce qui est évaluable est bien plus sa capacité à faire progresser et à organiser la connaissance que le chercheur peut avoir du phénomène qui l'intéresse que sa capacité à rendre compte d'un jeu de données aussi conséquent soit-il. Sur le simple objectif de l'utilisation du modèle comme artefact pour la réflexion, on peut se poser la question de savoir si l'évaluation du modèle a même un sens : est-ce que l'on évalue la feuille de papier qui nous permet de poser des hypothèses?

Enfin, au-delà de cette relation aux données qui diffère entre les deux approches de modélisation, d'autres propriétés souhaitables des modèles classiques ne sont simplement plus applicables aux modèles multi-agent. C'est le cas de la robustesse des modèles, c'est-à-dire leur capacité, lorsque l'on simule le modèle en partant de situations très proches, à obtenir des résultats très proches, autrement dit une forme de stabilité des résultats obtenus. On comprend très bien que c'est une propriété souhaitable du modèle lorsque l'on a des objectifs de prévision ou de prédiction concernant l'évolution d'un système. Cependant, dans le cas d'une modélisation compréhensive où l'objectif est d'apprendre des propriétés du système que l'on reconstruit in silico, le fait d'avoir, pour une partie des conditions expérimentales, des comportements très erratiques du système, loin de discréditer le modèle, nous apprend au contraire des éléments de son fonctionnement et nous permet même d'anticiper le fonctionnement du phénomène modélisé. Si dans certaines conditions le modèle est très instable, c'est une information très enrichissante sur le modèle et sur le phénomène considéré.

Pour conclure, il me semble que le pari de la modélisation agent en sciences sociales doit être fait par les chercheurs, en oubliant les fausses images d'une modélisation qui mutilerait ou réduirait la pensée sociologique ou d'un dispositif informatique destiné à capturer la connaissance sociologique dans un système expert; il s'agit bien plus d'une technique et d'une méthodologie permettant d'accompagner le chercheur en sciences sociales dans sa réflexion. 